\title{
OMNIS HOMO DE NECESSITATE EST ANIMAL: SIGNIFICACIÓN Y REFERENCIA VACÍA EN LA SEGUNDA MITAD DEL SIGLO $13^{1}$
}

\author{
Ana María Mora Márquez* \\ ana.maria.mora.marquez@gu.se
}

RESUMO $A$ questão "se uma elocução (palavra/termo/nome) perde seu significado com a destruição das coisas (quer dizer, as coisas significadas)" surge como uma questão sobre o valor-verdade de declarações com um termo vazio como sujeito, a saber, como um subproblema do sofisma "Se 'omnis homo de necessitate est animal'é verdade quando não há homem algum (=OHNEA)". Neste trabalho, trarei as discussões conforme elas se apresentam em "De signis" IV.2 de Roger Bacon, em "Quaestiones logicales", q. 2-3 de Peter John Olivi, no OHNEA de Boethius of Dacia, e no OHNEA de Anonymus Alani. Tais textos nos apresentam quatro modos diversos de nos relacionarmos com a questão sobre significação para OHNEA e, assim, com quatro posições diferentes sobre a relação entre significação e referência vazia. Usarei Anonymus Alani como o fio conductor de minha análise, inserindo outras posições onde tal se fizer relevante.

Palavras-chave Referência vazia, Lógica medieval, Semântica medieval, sofismas.

* Professor da Universidad de Gothenburg. Artigo recebido em 30/03/2014 e aprovado em 25/07/2014.

1 Este artículo es una versión corta y en español del cuarto capítulo de un libro (en preparación) sobre la noción de significación en el siglo 13. Mis agradecimientos van, por una parte, a Sten Ebbesen, Rodrigo Guerizoli, Christophe Grellard, Margareta Fredborg, Chris Martin y Paul Thom por sus valiosos comentarios sobre el contenido de este artículo; por otra parte a Ernesto Perini-Santos por su amable invitación a participar en este número de la revista "Kriterion" dedicado a la filosofía medieval y también por sus valiosos comentarios. 
ABSTRACT The question 'whether an utterance (word/term/name) loses its signification with the destruction of things (i.e. things signified)' is raised as a question about the truth-value of assertions with an empty term as a subject, namely as a sub-problem of the sophism 'Whether "omnis homo de necessitate est animal" is true when no man exists (=OHNEA)'. In this paper, I shall introduce the discussions as they are present in Roger Bacon's "De signis" IV.2, Peter John Olivi's "Quaestiones logicales", q. 2-3, Boethius of Dacia's OHNEA, and Anonymus Alani's OHNEA. These texts present us with four different ways of relating the question about signification to OHNEA, and therefore with four different positions regarding the relation between signification and empty reference. I shall use Anonymus Alani as the guiding thread of my analysis, inserting other positions where it is relevant.

Keywords Empty Reference, Medieval Logic, Medieval Semantics, Sophismata.

La cuestión 'Si una palabra (o término o nombre) pierde su significación con la destrucción de la cosa' (i.e., de la cosa que significa), que es en principio una pregunta sobre el valor semiótico de la palabra, está íntimamente ligada a la pregunta sobre el valor de verdad de las afirmaciones que tienen un término vacío como sujeto. Este lazo es evidente especialmente cuando la cuestión es un sub-problema del sofisma 'Si "Todo hombre es necesariamente animal" es verdadera cuando ningún hombre existe $\left(=O H N E A^{2}\right)$ '. ${ }^{3}$

En este artículo pretendo ilustrar la manera en la que la cuestión sobre la significación de las palabras se relaciona con la cuestión sobre la referencia vacía en algunas discusiones lingüísticas de la segunda mitad del siglo trece.

2 Edición en: A. de Libera, L. Gazziero, 2008.1, pp. 323-368. En este artículo seguiré la notación que usan los editores del sofisma.

3 Una lista de las diferentes versiones de este sofisma se encuentra en: S. Ebbesen, F. Goubier, 2010, pp. 21-61. En este sofisma también se discute sobre la modalidad de necesidad, sobre la composición que supone el uso del término 'est' y sobre la distribución que introduce el sincategorema 'omnis'. En este artículo me concentrare exclusivamente en la discusión sobre la relación entre la significación de cosas que no existen y el valor de verdad de las enunciaciones con sujeto vacío. Algunos estudios sobre modalidad, cuantificación y referencia vacía en el siglo 13 son: A. de Libera, 2009, pp. 179-233; id., 2002, pp. 201-237; id., 2002; id., 1997, pp. 177-200; id., 1991; L. Cesalli, A. de Libera, F. Goubier, 2013, pp. 305-336; L. Cesalli, 2007; S. Ebbesen, 1986, pp. 115-143; F. Goubier, 2000, pp. 37-70; E. Perini Santos, 2010; A. Tabarroni, 1993, pp. 185-201. Desde la década de 1230 se encuentran dubitationes acerca de OHNEA, en los tratados sincategoremáticos de Nicolás de París, Johannes Pagus, Pedro de España y Guillermo de Sherwood. Estas primeras discusiones, sin embargo, no se ocupan del problema de la referencia vacía, sino de los problemas de modalidad, cuantificación y composición; ver: Nicolás de Paris, "Syncategoremata", 1979; Johannes Pagus, "Syncategoremata", ed. Braakhuis, Vol. 1; Guillermo de Sherwood, "Syncategoremata", 1941, pp. 46-93; Pedro de España, "Syncategoremata", 1992. Sobre los sincategoremas en el siglo 13, ver: F. Goubier, "Les syncatégorèmes au XIlle siècle", 2003, pp. 85-113. 
Para este fin utilizaré los textos "De signis" (IV.2) de Roger Bacon OFM, ${ }^{4}$ las cuestiones 2-3 de las "Quaestiones logicales" de Pedro Juan Olivi OFM, ${ }^{5}$ y los sofismas OHNEA de los artistas Boecio de Dinamarca y Anonymus Alani (=An. A1.) Estos textos representan las cuatro posiciones principales con respecto a la relación entre estas dos cuestiones (i.e. significación y referencia vacía) y, por consiguiente, también representan cuatro aproximaciones diferentes a la noción de significación en este periodo de la lógica medieval: i) las palabras pierden su significación con la destrucción de las cosas que significan, de modo que 'OHNEA' no es ni verdadera ni falsa, salvo en el caso en que las palabras adquieran una nueva significación, de modo que 'OHNEA' sería falsa si ningún hombre existe (Roger Bacon); ii) las palabras no pierden su significación con la destrucción de las cosas que significan, sin embargo 'OHNEA' es falsa cuando ningún hombre existe (Boecio de Dinamarca); iii) las palabras no pierden su significación con la destrucción de las cosas, pero hay una ambigüedad en 'OHNEA', de modo que ésta es verdadera en un sentido, pero falsa en el otro (Pedro Juan Olivi); y iv) las palabras no pierden su significación con la destrucción de las cosas que significan y 'OHNEA' es siempre verdadera, sin importar si hay hombres o no (An. Al.). En las páginas siguientes me serviré del sofisma de An. Al. como hilo conductor de la exposición e insertaré las posiciones de los otros autores en los lugares donde sea pertinente.

\section{Anonymus Alani sobre 'omnis homo de necessitate est animal'}

An. Al. divide el sofisma OHNEA en tres problemas (problemata): a) si la necesidad de la composición que incluye la afirmación requiere la existencia necesaria del sujeto y del predicado; ${ }^{6}$ b) si la afirmación 'OHNEA'

\footnotetext{
Cf. Roger Bacon, "De signis", 1978, pp. 75-136.

5 Cf. Pedro Juan Olivi, "Quaestiones logicales", 1986, pp. 335-388.

6 Entiéndase, la existencia de los significados de la expresión nominal y de la expresión predicativa. Nótese que este problema pregunta literalmente si la necesidad de la composición lingüística implica la existencia 'necesaria' de los términos y no si la composición implica 'necesariamente' la existencia de los términos. Algunos autores de la época, como Boecio de Dinamarca, sostienen de hecho que una enunciación modal de necesidad sólo puede ser verificada por cosas cuya existencia es necesaria, de modo que toda enunciación modal de necesidad cuyo sujeto es una cosa material es, por consiguiente, falsa. Otros autores, como Roberto Kilwardby y Guillermo de Sherwood, proponen, por ejemplo, una distinción entre necesidad esencial y necesidad accidental que resulta en condiciones de verdad que incluyen enunciaciones de necesidad cuyo sujeto es algo contingente. Sobre la modalidad en la lógica medieval en general, ver S. Knuuttila, 1993. Sobre Boecio de Dinamarca, ver: S. Ebbesen, 2000, pp. 145-158; id., 2005, pp. 235-250.
} 
es verdadera o falsa; y c) si el término pierde su significado con la destrucción de la cosa que significa. ${ }^{7}$

La discusión comienza con la introducción de los argumentos que prueban de manera directa que la necesidad de la composición lingüística, que en el caso de 'OHNEA' es representada por el término 'est', implica la existencia necesaria del sujeto y del predicado en por lo menos un individuo. ${ }^{8}$ Uno de estos argumentos es el siguiente:

a) En una afirmación, la composición es a los términos como la forma es a la materia;

b) pero la forma necesaria (i.e. la composición necesaria) no puede informar la materia contingente (e.g. los términos 'homo' y 'animal');

c) por lo tanto, la necesidad de la composición implica la existencia necesaria de los términos (i.e., de 'homo' y de 'animal'). ${ }^{9}$

En otras palabras, sólo hay composición necesaria si la existencia de por lo menos un individuo compuesto de hombre y animal es necesaria; pero ningún compuesto material existe de manera necesaria; de ahí que si por hipótesis se supone que ningún hombre existe, que es una hipótesis plausible, entonces la afirmación de necessitate 'OHNEA' es falsa.

Acto seguido, el autor anónimo desecha el cuantificador universal 'omnis' y la modalidad de necesidad y procede a preguntar si la afirmación indeterminada 'homo est animal' es verdadera o falsa cuando ningún hombre existe; si es verdadera, entonces algún tipo de necesidad parecería estar implicado en la predicación esencial, que puede ser el tipo de necesidad que está detrás de la verdad de la afirmación universal y de necessitate 'OHNEA'.

La equivalencia entre predicación esencial, cuantificación universal y necesidad de la composición se basa directamente en los primeros dos sentidos de predicación per se que Aristóteles discute en "Analíticos Segundos" I.4, es decir, i) cuando se atribuyen al sujeto partes de su definición, e.g. 'animal' a

7 Cf. An. Al., OHNEA, ed. Libera et Gazziero, pp. 332: "Circa istud sophisma plura fuerunt quaesita, sed tantum tria prosecuta. Primum est utrum necessitas compositionis requirat necessario existentiam extremorum. Secundum est de veritate et falsitate primae propositionis. Tertium utrum terminus cadat a suo significato, quando res desinit esse".

8 Otra cuestión intímamente relacionada con la nuestra es si deben existir por lo menos tres individuos del término común para que la cuantificación universal tenga sentido. Este problema se discute, por ejemplo, en el sofisma 'Omnis phoenix est animal'. Una lista de las versiones de este sofisma se encuentra en: S. Ebbesen, F. Goubier, pp. 362-370; un análisis sobre el mismo sofisma se encuentra en: L. Cesalli et al., 2013.

9 An. Al., OHNEA, ed. Libera et Gazziero, pp. 334: "Item. Compositio sic se habet ad extrema sicut forma ad materiam; sed in materia corruptibili et non necessaria non potest esse forma necessaria; ergo in extremis corruptibilibus non potest esse compositio necessaria; ergo ad necessitate compositionis requiritur necessitas extremorum". El autor propone 10 argumentos más que sostienen la misma posición; cf. An. Al., OHNEA, ed. de Libera et Gazziero, pp. 332-336. 
'hombre'; o ii) cuando se atribuyen a un sujeto un par de opuestos que le son coextensivos, e.g., 'par/impar' a 'número'. ${ }^{10}$ Aristóteles sugiere que estos dos tipos de predicación per se son universales y necesarios. ${ }^{11}$ En otras palabras, si la extensión de A está por definición incluída en la extensión de B (e.g. la extensión de 'hombre' en la extensión de 'animal'), o si por definición A y B son coextensivos (e.g. la extension de 'par/impar' que es igual a la extension de 'número natural'), entonces 'A es B' es necesaria y universal.

Uno de los cuatro argumentos que prueban que 'homo est animal' es falsa cuando ningún hombre existe es:

a) Una enunciación es verdadera porque representa una composición o división mental verdadera (cf. Arist., Int. 1);

b) pero el intelecto compone y divide cosas que existen y no sólo sus conceptos (de otro modo toda composición mental sería falsa porque todos los conceptos en cuanto conceptos son diferentes entre sí);

c) por hipótesis ningún hombre existe;

d) por lo tanto no puede haber una composición mental verdadera entre el hombre y el animal (por b);

e) por lo tanto 'homo est animal' no es verdadera (por a);

f) por lo tanto 'homo est animal' es falsa (por el principio de bivalencia);

g) por lo tanto 'omnis homo est animal' y 'omnis homo de necessitate est animal' son también falsas, supuestamente porque el antecedente de la inferencia 'per se, ergo universal, ergo necesario' no se da. ${ }^{12}$

Con respecto a este argumento, podemos notar por lo menos dos problemas. Por una parte, la inferencia 'per se, ergo universal, ergo necesario' supone que A y B no sean términos vacíos; pues la demostración de atributos en el contexto de los "Analíticos Segundos" supone la existencia del sujeto, de modo que cuando se asume por hipótesis que ningún hombre existe, la

10 Cf. Arist. Lat. APo I.4, ed. Hamesse, 314, pp. 38-41: "Per se primo sunt quaecumque in ratione dicente quod quid est insunt [...] per se secundo sunt, quaecumque insunt ipsis [...] ut propria passio per se inest subiecto [...] Item per se sunt, quae non dicuntur de quodam alio subiecto, ut substantia. Item per se sunt, propter quod inest alicui aliquid, tamquam per causam. Hic habemus, quod quattuor sunt modi dicendi per se, quorum primus et secundus tantum ingrediuntur demonstrationem". Sobre la teoría aristotélica de la predicación, ver: A. Bäck, 2000, ch. 6.

11 Cf. APo I.4.

12 An. Al., OHNEA, ed. de Libera et Gazziero, p. 337: "Quia veritas est in oratione sicut in signo. Significat enim oratio veritatem quae est circa compositionem et divisionem intellectus componentis vel dividentis; intellectus autem non componit intentiones quas habet penes se de rebus extra ita quod dicat quod haec est illa; sic enim esset omnis compositio eius falsa; et etiam intelligens et iudicans non iudicat de ipsis intentionibus vel speciebus sed de rebus quarum sunt; componit ergo ad invicem <res>; res autem vere componere non potest nisi res ipsae sint; ergo ad hoc quod compositio designata per hanc "omnis homo est animal" sit vera, oportet quod omnis homo sit; cum ergo hominem esse non sit necessarium, non erit haec necessaria "omnis homo est animal"; ergo nec erit haec vera "omnis homo de necessitate est animal". 
inferencia en cuestión es por lo menos irrelevante o bien su relevancia bajo esta hipótesis debería ser justificada. Por otra parte, el paso de e) a f) supone el uso del principio de bivalencia, principio que por demás no parece aplicar a las enunciaciones indefinidas, como 'homo est albus' y 'homo non est albus', que pueden ser verdaderas al mismo tiempo. ${ }^{13}$ Así, resulta por lo menos problemático asumir que el sujeto es vacío y aplicar al mismo tiempo la inferencia 'per se, ergo universal, ergo necesario' y el principio de bivalencia.

Los argumentos que conceden 'OHNEA' como verdadera presentan problemas similares. Llamemos al más importante de estos argumentos, que An. Al. concederá en su solución del sofisma, el argumento A. Este argumento por reducción al absurdo puede ser reconstruído de la manera siguiente:

a) En 'OHEA', 'homo' tiene suposición personal; ${ }^{14}$

b) toda enunciación cuyo término sujeto tiene suposición personal implica tres relaciones: i) una entre el término sujeto y el predicado: ii) otra entre el término sujeto y sus supuestos; y iii) una última entre el predicado y los supuestos del sujeto;

c) la enunciación de este tipo afirma estas tres relaciones;

d) su negación niega la primera y la tercera, pero afirma la segunda;

e) por lo tanto, en la negación 'Non OHEA', 'homo' pone todos sus supuestos como sujeto de atribución, negando al mismo tiempo que el atributo 'animal' se aplique a todos ellos;

f) por lo tanto por lo menos un supuesto del hombre, e.g. Platón, no tiene una relación con 'animal';

g) por lo tanto 'Plato non est animal' es verdadera;

h) por lo tanto 'Plato non est homo' es verdadera (porque homo $={ }_{d}$ animal rationale);

i) pero 'Plato est homo' es verdadera (por d.);

j) entonces 'non OHEA' implica tanto 'Plato est homo' como 'Plato non est homo';

k) por lo tanto 'non OHEA' es imposible;

13 Cf. Arist., Int. 7.17b29-32.

14 Más exáctamente, tiene suposición personal confusa. Según, e.g., Pedro de España, Tractatus, ed. L.M. de Rijk, 1972, la suposición de un término es personal cuando el término común se toma por sus individuos. Cf. Pedro de España, "Tractatus", ed. De Rijk, p. 82: "Personalis suppositio est acceptio termini communis pro suis inferioribus. Ut cum dicitur 'homo currit', iste terminus 'homo' supponit pro suis inferioribus". Ahora bien, la suposición es personal confusa cuando el término se toma por varios individuos (i.e. todos sus individuos), por medio del signo universal 'omnis'. Cf. Pedro de España, "Tractatus”, ed. De Rijk, 82, p. 82: "Confusa suppositio est acceptio termini communis pro pluribus mediante signo universali. Ut cum dicitur 'omnis homo est animal', iste terminus 'homo' mediante signo universali tenetur pro pluribus, quia pro quolibet suo supposito". Sobre la teoría medieval de la suposición en general, ver Catarina Dutilh Novaes, 2011, pp. 1229-1236. 
1) por lo tanto 'OHEA' es necesaria;

m) por lo tanto 'OHNEA' es verdadera. ${ }^{15}$

Este argumento, sin embargo, asume de nuevo la existencia de algún supuesto del hombre, lo que es negado por hipótesis. Parece entonces que ambos argumentos, tanto el que prueba la falsedad de 'OHNEA' como el que prueba su verdad, son problemáticos si se toma por hipótesis que ningún hombre existe.

La naturaleza paradójica del sofisma OHNEA depende en gran medida de las consecuencias lógicas de aplicar los sincategoremas 'omnis' y 'necessario' a una composición que toma como sujeto un término vacío, dado que las propiedades lógicas de estos sincategoremas parecen suponer en cierta medida la existencia de inviduos de los términos que afectan. Ahora bien, que un término no tenga supuestos equivale a no tener significación? Es así como se establece el lazo entre la cuestión de la verdad de 'OHNEA' y la pregunta sobre la permanencia de la significación de un término cuando la cosa que significa es destruída. An. Al. procede entonces a discutir esta última pregunta, pero antes de presentarnos su solución, rechaza tres respuestas similares a las que encontramos en los autores Roger Bacon, Boecio de Dinamarca y Pedro Juan Olivi.

\section{Roger Bacon en "De signis" IV.2}

En "De signis", Roger Bacon propone la pregunta sobre la permanencia de la significación de una palabra - si la palabra sigue siendo significativa después de su imposición arbitraria - y procede al análisis del caso problemático en el que la cosa significada deje de existir. El argumento de Bacon que prueba que una palabra pierde su significación con la destrucción de la cosa que significa es:

a) la significación es una relación entre una palabra y una cosa;

b) toda relación se destruye con la destrucción de uno de sus términos;

15 An. Al., OHNEA, ed. Libera et Gazziero, p. 338: "Item. Quicumque negat eam, sive sit homo sive non, idem affirmat et negat; ergo sua negativa est impossibilis. Probatio antecedentis: terminus habens personalem suppositionem supponit suum significatum pro appellato vel appellatis, [...]; ergo per dictum tuum qui negat eam removet esse animal ab appellatis 'hominis'; ergo cum ista negativa designet tres habitudines sicut et quaelibet negativa cuius subiectum habet personalem suppositionem, unam praedicati ad subiectum, aliam subiecti ad suum suppositum, tertiam praedicati ad suppositum subiecti, prima designabitur in ea negative, secunda affirmative et tertia negative. [...] Semper in propositione autem affirmativa designantur esse tres dictae habitudines affirmativae; tu ergo in tua negativa qua explicite negas esse animal de homine affirmas implicite esse hominem de eius supposito et removes animal de eodem, quod idem est implicite ac si explicite diceres quod ipse est homo et non est animal. [...] Ergo patet quod per tuam negativam dicis quod ipse est homo et non est homo. Et sic idem affirmas et negas". 
c) por lo tanto si la cosa es destruída, la relación de significación de la palabra se destruye también. ${ }^{16}$

Bacon añade a su argumento un ejemplo que es reproducido en el sofisma $O H N E A$ de An. Al. - la señal de vino en una tienda, que no tiene significación alguna si el vino de la tienda se terminó. Si esta señal puede aún ser interpretada como el signo de algo, dice Bacon, sería de una imagen del vino en la mente de aquellos que todavía no saben que el vino de la tienda se terminó, y ésto gracias a la reimposición de la señal a esa imagen. ${ }^{17}$ Este extraño ejemplo, en el que Bacon nos dice que el signo de algo que no existe es reimpuesto a una imagen de la cosa, tiene una desafortunada consecuencia, pues nos fuerza a suponer que los compradores que no saben que en la tienda no hay vino reimponen el signo de manera inconsciente - puesto que ellos no saben que en la tienda no hay vino, tampoco pueden reimponer de manera consciente la señal a la imagen del vino. Bacon añade que lo mismo pasa en el caso de las palabras, de modo que 'Sócrates', que fue impuesta para significar una persona que ya no vive, perdió su significación original cuando 'Sócrates' murió, y es ahora usada de acuerdo a una significación por reimposición.

El franciscano explica los mecanismos de la reimposición estableciendo dos tipos de imposición: una primera imposición cuando una palabra es dada por primera vez a algo como su nombre (sobre el modelo del bautizo); y una

16 Roger Bacon, "De signis" IV.2, ed. Fredborg et al., p. 128: "Item, corrupto uno extremorum relative oppositorum licet non corrumpatur alterum secundum substantiam eius, corrumpitur tamen relatio et habitudo unius ad aliud, ut corrupta substantia Socratis, qui fuit filius Platonis, Plato licet vivat, tamen paternitas non manet in Platone. Similiter in omnibus. Quare re significata corrupta licet substantia vocis maneat non tamen ratione signi". El extensionalismo radical de Bacon implica, por un lado, el rechazo de las nociones de 'esse habitualis' y de 'esse essentiae' que usan otros autores con el fin de dar cierta estabilidad ontológica a los significados de las palabras; por otro lado, también implica la consideración de la cópula 'est' como indicador de una relación de inherencia entre cosas que existen. El extensionalismo de Bacon podría estar relacionado con su participación en ciertos debates de carácter teológico, uno de los cuales gira en torno a la tesis de e.g. Ricardo de Cornwall, según la cual 'homo' es unívoco con respecto a Jesús y a su cadáver durante los tres días anteriores a la resurrección. Bacon rechaza esta tesis en el "Compendium theologiae" y afirma que el cadáver de Jesús sólo puede llamarse 'homo' después de una reimposición del nombre, de modo que cuando se aplica a Jesús vivo y a Jesús muerto, 'homo' es un nombre equívoco. Sobre este debate, ver: A. de Libera, 1991, pp. 85-120; id., 1981, pp. 193-221; sobre Bacon y la equivocidad, ver: T. S. Maloney, 1984, pp. 85-112.

17 Roger Bacon, "De signis" IV.2, ed. Fredborg et al., p. 128: "Item, hoc videmus in signis aliis, cum enim non <est> vinum in taberna et emptores vini hoc <sciunt>, iam circulus vini non recipitur apud eos pro signo, et ita est in aliis. Quapropter corrupta re, cui facta est impositio, non remanebit vox significativa. Si dicatur: bene accidit quod vinum non sit in taberna, et tamen emptores videntes circulum quaerunt vinum propter circulum, ergo circulus adhuc est eis signum, quare similiter hoc dicendum est quod scientibus, quod non sit ibi vinum, circulus qui fuit signum verum et veri vini iam cadit apud eos et secundum veritatem a ratione signi. Sed ignorantes vinum non esse et putantes esse in taberna, cum non sit, videntes circulum aestimant circulum significare sibi vinum et renovant rationem signi in eo, ita quod apud imaginationem suam constituunt circulum esse signum vini aestimati ab eis et imaginati, et sic fit circulus signum novum. Sed cecidit a ratione prima significandi, quam habuit respectu vini veri. Et similiter potest esse de vocibus". Cf. An. Al., OHNEA, ed. Libera et Gazziero, p. 350. 
segunda imposición que tiene lugar en la mente, cuando los hablantes piensan en algo sobre lo que quieren hablar y le reimponen palabras que según la primera imposición habían sido dadas a otra cosa. ${ }^{18}$ Fiel al hincapié franciscano en la voluntad humana, Bacon explica que la reimposición es posible porque el acto de imposición es dirigido por la voluntad, de modo que el hablante puede tener la voluntad de reimponer la palabra al objeto de su pensamiento y expresar esta nueva imposición en el lenguaje. ${ }^{19}$ Esto es lo que sucede cuando hablamos de un hombre que ya no existe, como Sócrates, o cuando llamamos 'hombre' la pintura de un hombre. El uso cotidiano del lenguaje implica un largo encadenamiento de reimposiciones que, sin embargo, toma lugar en contextos enunciativos, de modo que los actos de reimposición pasan desapercibidos, ya que no hay un lapso perceptible de tiempo entre la reimposición de las palabras y su uso en una enunciación. ${ }^{20}$

Bacon afirma también que cualquier enunciación en acuerdo con un acto de reimposición es necesariamente verdadera o falsa, pues necesariamente indica algo que es el caso o que no es el caso sobre el objeto de la reimposición. Ahora bien, si la enunciación no siguiera un acto de reimposición, no podría indicar de ninguna manera algo verdadero o falso, porque sus términos no tendrían significación: ${ }^{21}$ es decir que si los términos de la enunciación no tienen significación, simplemente no hay enunciación. Se podría afirmar entonces que en el caso de que ningún hombre exista, si el hablante no reimpone los términos 'homo' y 'animal' a algo más, éstos no tienen significación alguna, de modo que la enunciación 'OHNEA' no es ni verdadera ni falsa, de hecho no es ni siquiera una enunciación..$^{22}$ La noción de reimposición, sin embargo,

18 Roger Bacon, "De signis" IV.3, ed. Fredborg et al., p. 130: "Ad horum ergo evidentiam potest quaeri de modo imponendi duplici an sit, ut tactum est, et dicendum quod sic. Unde unus est modus imponendi sub forma impositionis vocaliter expressa et assignata rei sicut imponuntur nomina infantibus et aliis rebus. $<$ Sed alius est modus imponendi quid fit apud solum intellectum considerantem ens $>$ vel non ens, de quo vult aliquid enuntiare, vel quod de alio vult enuntiare, et sic imponit nomen".

19 Roger Bacon, "De signis" IV.3, ed. Fredborg et al., p. 130: "Quod autem hoc sit possibile manifestum est, quia positio est ad placitum, ergo secundum quod placet homini, potest in mente sua dare vocem rei vel exprimere impositionem vocaliter. Sic potest de omnibus aliis, de quibus cogitat et quae vult".

20 Roger Bacon, "De signis" IV.3, ed. Fredborg et al., pp. 131-132: "Si dicatur quod tunc avertemus impositiones istas, sed nullus quidem avertit, quando imponit, dicendum quod ubi non diligenter consideraret non bene averteret rationem et horam imponendi <nisi fieret impositio sub forma imponendi> vocaliter expressa quia haec est prima et principalis et consueta in rebus et linguis, [...] Et haec est prima causa latentiae. [...] Tertia causa est quod non fiunt multum istae impositiones, nisi simul fiant enuntiationes de illis terminis, ita quod non est tempus sensibile nec minimum inter impositionem et enuntiationem, [...]".

21 Roger Bacon, "De signis" IV.2, ed. Fredborg et al., p. 129: "Et dicendum est quod si fiat impositio, necesse est quod talis oratio sit propositio et sit vera vel falsa, et quando volumus significare verum vel falsum, necessario prius imponimus et transsumimus vocem ad significandum".

22 Roger Bacon, "De signis" IV.2, ed. Fredborg et al., p. 129: "Sed si non fieret impositio aliqua, certum est quod talis oratio esset propositio neque vera neque falsa immo esset non significativa; ab una enim parte non significativa fit tota oratio non significativa, et [per] hoc patet". 
asegura que los términos de todas nuestras enunciaciones tengan significación, porque siempre que queremos afirmar algo sobre algo reimponemos las palabras que usamos a las cosas de las que queremos hablar.

La desafortunada consecuencia de la solución de Bacon es la de tener una enunciación diferente en cada pronunciación, ${ }^{23}$ con lo que resultaría difícil explicar la posibilidad de la comunicación linguística. ${ }^{24}$ Así, 'Socrates est homo' es una enunciación cuando Sócrates está vivo, y otra enunciación cuando Sócrates ya no vive y cuando tanto 'homo' como 'Socrates' han sido reimpuestas a, e.g., una imagen mental o una pintura de Sócrates, de modo que en ambas enunciaciones 'Sócrates' es equívoco con respecto al hombre vivo y al hombre en la pintura. Nótese entonces y para terminar que, estrictamente hablando, para Bacon no hay términos vacíos, porque una vez que un término es usado en un contexto enunciativo es porque fue (re)impuesto a algo. El problema en el caso de Bacon es entonces más un problema de equivocidad que un problema de referencia o de significación vacías.

\section{Boecio de Dinamarca y su sofisma OHNEA}

Otra posición consiste en negar que el término pierda su significación con la destrucción de la cosa que significa, rechazando al mismo tiempo 'OHNEA' como falsa. Boecio de Dinamarca es sin duda el autor que mejor representa esta posición. El maestro danés divide su discusión del sofisma OHNEA en cuatro problemas, de los cuales sólo dos nos conciernen: i) si 'OHNEA' es verdadera cuando ningún hombre existe; y ii) si los términos pierden su significado con la destrucción de las cosas.

Con respecto al primer problema, Boecio afirma que la enunciación 'OHNEA' es falsa por causa de su modalidad; pues el hombre y el animal son cosas contingentes y susceptibles de cambio - son esencias que informan la

23 Roger Bacon, "De signis" IV.2, ed. Fredborg et al., pp. 129-130: "Si obiciatur quod Aristoteles in Praedicamentis < dicit> quod oratio remanet vera vel falsa, et mutatur de vera vel falsa, ut 'Socrates sedet', haec est vera, dum sedet, eo modo surgente haec eadem est falsa, et ita similiter 'Socrates est', [...] Sed si eadem est oratio, idem est subiectum, ergo idem est significatum per vocem subiecti, [...] Dicendum est quod ibi loquitur de praedicatis accidentalibus, et quae possunt separari a subiecto existente, ita quod dictio 'Socrates' remaneat significativum (significativa an scribendum?) eius, quod prius significabat; [...] Sed si accipiatur praedicatum quod inest per se et essentialiter ut 'Homo est <homo>', 'Homo est' et huiusmodi, non est possibile quod fiat mutatio eiusdem orationis a vero in falsum, sed erit diversa et diversa oratio, et ideo diversum subiectum et diversum significatum subiecti et accipietur aequivoce. Ut dum Socrates est, haec est vera 'Socrates est' et 'Socrates' significat vivum. Dum vero Socrates non est et dicatur haec oratio 'Socrates est', haec est falsa, sed non est oratio eadem quae prius [...]".

24 Bacon, sin embargo, no parece ser consciente de lo problématico de su solución. 
materia destruíble - de ahí que el hombre y el animal no puedan causar una verdad necesaria. ${ }^{25}$ Este argumento, sin embargo, es de carácter metafísico.

Boecio también presenta, y concede, el siguiente argumento:

a) Hay composición lingüística, composición mental y composición real;

b) la verdad de la composición lingüística depende de la verdad de la composición mental, porque la verdad de lo que es posterior depende de la verdad de lo que es anterior;

c) de la misma manera, la causa de la composición mental es la composición real - 'el soporte y la causa de toda verdad posterior';

d) por lo tanto si no hay composición real, ni la composición mental ni la lingüística pueden ser verdaderas;

e) pero es imposible que haya composición real del hombre y el animal cuando ningún hombre existe;

f) por lo tanto cuando ningún hombre existe es imposible que cualquier composición lingüística que envuelva el hombre y el animal sea verdadera. $^{26}$

Boecio rechaza entonces 'OHNEA' como falsa por razones metafísicas, pero sus razones lógicas se limitan a mostrar que 'OHNEA' no puede ser verdadera, siendo lo suficientemente cuidadoso como para no concluír que es falsa. ${ }^{27}$

Algunas páginas después, Boecio afirma que aunque la significación de las palabras sea la misma sin importar la existencia o no existencia de las cosas, la significación no es la causa de la verdad, sino el hecho de que las cosas sean o no de una cierta manera. Boecio relega así la noción de significación a un campo en el que le queda poco o ningún pápel que jugar en la cuestión

25 Boecio de Dinamarca, Omnis homo etc., ed. Ebbesen, p. 7: "Cum ergo homo et animal sint res transmutabiles et corruptibiles et nihil sit in eis incorruptibile nisi materia prima, [...], secundum quam materiam non verificatur ista propositio 'homo de necessitate est animal', sequitur quod ex hiis rebus homo et animal nulla causatur veritas intransmutabilis nec etiam necessaria".

26 Boecio de Dinamarca, Omnis homo etc., ed. Ebbesen, p. 3: "Triplex invenitur compositio, scilicet compositio quae est in re ipsa, compositio quae est in intellectu, et compositio quae est apud sermonem, ita quod in istis semper veritas quae est in posteriori compositione est ex veritate prioris compositionis tamquam ex causa sua. [...] quomodo erit compositio vera apud intellectum nisi consimilis sit compositio in re? Si enim sit in re divisio et apud intellectum compositio, erit falsa compositio intellectus. Ex hoc arguitur sic: Sicut se habet compositio sermonis ad compositionem intellectus, sic se habet compositio intellectus ad compositionem rerum; sed compositio sermonis non potest esse vera nisi sit vera compositio intellectus ex qua ipsa est; ergo nec potest esse vera compositio intellectus nisi sit talis compositio in re. Sed nullo homine existente animal homini non componitur; ergo nec intellectus nec sermo componens animal homini potest esse verus, cum deficiat compositio quae est in re, quae est fundamentum et causa cuiuslibet veritatis posterioris, scilicet intellectus et sermonis".

27 Que es, sin embargo, lo que hace el argumento presentado por An. Al. Ahora bien, que 'OHNEA' no pueda ser verdadera no implica que sea falsa, porque bien podría no tener valor de verdad - no ser ni verdadera ni falsa. 
de la verificación proposicional. ${ }^{28} \mathrm{El}$ hecho de que las palabras conserven su significación después de la destrucción de las cosas no tiene nada que ver con el hecho de que las enunciaciones que contienen estas palabras como sujetos sean verdaderas o falsas, de modo que Boecio desliga la significación que sigue a la primera imposición de una palabra de la cuestión sobre la verdad o falsedad de las enunciaciones.

\section{Pedro Juan Olivi ${ }^{29}$ y su quaestio logicalis 3}

Finalmente, está la posición según la cual los términos no pierden su significación con la destrucción de las cosas y que establece una distinción entre ser disposicional (esse habituale) y ser actual (esse actualis), que resuelve la cuestión sobre la verdad de 'OHNEA'. Pedro Juan Olivi menciona esta distinción en su respuesta a la cuestión 'Si la existencia actual de los sujetos y los predicados sigue de la verdad de las proposiciones necesarias afirmativas' ("Quaestiones logicales”, q. 3). ${ }^{30}$ Olivi presenta su solución con base en la posición 'común y razonable' según la cual 'esse' no siempre pretende expresar la existencia actual, sobre todo cuando se usa como tercero adyacente, en cuyo caso normalmente lo que se quiere expresar es la relación y coherencia esenciales entre el sujeto y el predicado. ${ }^{31}$ Así, 'homo est animal' pretende expresar que la noción de animal es una parte esencial de la noción de hombre y que, por consiguiente, en cierta forma el hombre y el animal son la misma cosa. ${ }^{32}$

El tipo de ser representado por 'est' cuando es tercero adyacente, dice Olivi, es llamado 'ser disposicional' o 'ser de esencia'. ${ }^{33}$ Pero vale aclarar que, según Olivi, el ser disposicional y el ser actual no implican una multiplicación del inventario ontológico del mundo, sino una doble modalidad de la

28 Sobre la verificación proposicional en Boecio de Dacia, ver: A.M. Mora-Márquez, 2014, pp. 23-48.

29 Sobre la teoría de la significación de Olivi, ver: A. M. Mora-Márquez, 2011, pp. 150-164; sobre Olivi en general, ver: A. Boureau \& S. Piron (eds.), 1999.

30 Pedro Juan Olivi, QL, q.3, ed. Brown, p. 344: "Post hoc quaeritur: $<A n>$ ad veritatem propositionum necessariarum et affirmativarum sequatur actualis entitas subiecti et praedicati? Utpote: An sequatur 'Homo est animal, ergo est actu'?"

31 Pedro Juan Olivi, QL, q.3, ed. Brown, p. 344: "Respondeo: Quidam dixerunt quod ad veritatem huiusmodi propositionum praeexigitur praesuppositio actualis existentiae subiecti, [...] Alii vero communius et rationabilius dicunt quod per verbum essendi quando sumitur sub sola ratione copulativae duorum non semper intendimus significare exsistentiam actualem, sed potius eorum identitatem sive essentialem habitudinem et cohaerentiam".

32 Pedro Juan Olivi, QL, q.3, ed. Brown, pp. 344-345: “Unde cum dicimus 'Homo est animal' non intendimus dicere quod homo sit actu animal, sed solum quod animalis ratio sic cohaeret rationi hominis quod est pars eius, et aliquo modo sunt idem".

33 Pedro Juan Olivi, QL, q.3, ed. Brown, p. 345: "Et 'esse' sic acceptum per modum solius copulae [...] vocatur a quibusdam esse habituale vel esse essentiae". 
aproximación cognitiva a la esencia; pues el ser disposicional corresponde a la esencia sólo en la medida en que ésta es susceptible de entenderse sin su existencia actual. ${ }^{34}$

Olivi procede a introducir otra distinción que concierne la aproximación cognitiva a la esencia que tiene existencia actual, a la que nos podemos aproximar según un modo nominal (o esencial) o según un modo verbal. El modo nominal se da cuando consideramos la definición de un ser actual, pero separamos esta definición de la actualidad de su existencia. El modo verbal se da cuando expresamos con un verbo que la esencia tiene existencia actual. ${ }^{35}$ Así, incluso en el caso en el que algunos individuos del hombre existan, en la frase definicional 'homo est animal', 'homo' sólo indica su esencia y no su existencia. De otra parte, el uso del verbo 'est' en 'homo est' hace que 'homo' indique de manera explícita la existencia actual de algún individuo del hombre. De este modo, de acuerdo con la manera de expresar y de entender la esencia del hombre, 'homo est animal' no es equivalente a 'homo est actu animal'36 (en esta última la existencia del hecho significado se hace explícita por medio de 'actu'). 'Homo est animal' es entonces verdadera, porque, normalmente, con esta expresión se pretende hablar del ser de esencia del hombre. Pero si se hace explícito que se pretende hablar de su existencia actual, como cuando se añade 'actu', en este caso la afirmación sería falsa.

\section{Anonymus Alani y su solución del sofisma OHNEA}

Con el fin de rechazar tres posiciones similares a las que vengo de exponer, el autor anónimo establece dos supuestos: i) la existencia no se incluye en el

34 Pedro Juan Olivi, QL, q.3, ed. Brown, p. 345: "Ad tertium dupliciter respondetur: Primo, quod esse habituale differt ab esse actuali, non quidem realiter, quasi sint diversa esse realia in rebus exsistentia, sed solum differunt secundum diversas rationes sive modos, quia esse habituale convenit rei prout est intelligibilis absque cointelligentia suae actualis exsistentiae. Et ideo prima ratio sive primus modus essendi potest ab intellectu accipi absque secunda. Sicut enim differt ratio essentiae a ratione suae actualis exsistentiae absque hoc quod inter se habeant aliquam differentiam vel compositionem realem, sic differt esse habituale ab esse actuali, quia primum esse respicit solam rationem essentiae et suorum essentialium in quantum se tenent cum ratione exsistentiae actualis".

35 Pedro Juan Olivi, QL, q.3, ed. Brown, pp. 345-346: "Secundo, dicitur quod idem esse actuale potest dupliciter accipi: Uno modo per modum nominalem et per modum quidditatis, sicut fit cum intelligitur ratio cuiuscumque esse actualis, quamvis ipsum non sit actu, aut quamvis non cogitetur ipsum esse in actu. Et hoc modo sumitur esse habituale terminorum essentialium, quia cum quis cogitat rationem hominis aut animalis vere cogitat rationem entis et rationem sui esse sibi essentialis, per modum tamen quidditatis cogitando scilicet quod dicunt et quas rationes in se includunt, quamvis ex hac non sciantur nec fore credantur esse in actu. Alio modo sumitur esse actu per modum verbalem prout scilicet scitur vel dicitur actualiter esse in rebus".

36 Pedro Juan Olivi, QL, q.3, ed. Brown, p. 346: "A primo autem modo sumendi non sequitur iste secundus modus. Et ideo secundum modum intelligendi et enuntiandi non est <idem $>$ dicere 'Homo est animal' quam dicere "homo est actu animal'." El editor suple < aliud >, pero el sentido del pasaje requiere <idem $>$. 
valor semiótico de una palabra; y ii) hay una diferencia entre el ser de esencia y el ser de existencia. ${ }^{37} \mathrm{El}$ primer supuesto, que por demás es compartido por Boecio de Dinamarca, deriva del primer capítulo del "De interpretatione", donde Aristóteles dice que los términos nominales (onomata) y los términos atribuídos (rhêmata), cuando se toman en sí mismos no afirman nada; deriva también del capítulo 3 del mismo tratado donde el Estagirita nos dice que los terminos atribuídos (rhêmata) no afirman existencia o no-existencia, cuando están por fuera de una enunciación. ${ }^{38} \mathrm{El}$ segundo supuesto, según An. Al., es un corolario del primero, porque si las palabras no significan la existencia, entonces sólo significan la esencia, que es aquello mismo que la definición significa; ${ }^{39}$ pero las palabras no significan la existencia, porque cuando el intelecto entiende la esencia de una cosa, no entiende necesariamente al mismo tiempo su existencia. ${ }^{40}$

An. Al. se sirve de estos dos supuestos en su rechazo de tres posiciones similares a las de Bacon, Boecio y Olivi, aunque, como veremos, ninguna de las posiciones que rechaza corresponde de manera exacta a las posiciones de estos autores.

Contra la posición según la cual los términos son equívocos con respecto a la existencia y a la no-existencia (que encontramos, e.g., en la renuencia de Bacon de aceptar "Socrates est homo" como verdadera simpliciter) An. Al. nos dice que esto va en contra de la afirmación en las "Categorías" (Arist. Cat., 5.4a21-b13), según la cual una enunciación pasa de ser verdadera a ser falsa por causa de una alteración del sujeto, con el sujeto lógico permaneciendo igual. ${ }^{41}$ Para Bacon, sin embargo, esta afirmación de las "Categorías" sólo es válida en el caso de la predicación accidental. A esto, An. Al. responde que

37 An. Al., OHNEA, ed. Libera et. Gazziero, p. 344: "In his autem rationibus et in aliis sequentibus accipiam duas suppositiones. Prima est quod nomen de vi vocis non significat rem esse, cuius est ipsum nomen etiam si res sua sit. Secunda est quod esse existentiae et esse essentiae non sunt idem".

38 An. Al., OHNEA, ed. Libera et Gazziero, p. 344: "Et ne suppositiones videantur impossibiles possunt confirmari. Prima sic. Dicit Aristoteles primo Peri hermeneias capitulo primo: '[...] Nomina igitur et verba $<$ con> similia sunt intellectui sine compositione et divisione ut "homo" vel "album", quando non additur aliquid; neque adhuc verum vel falsum est [...]' [...] Item. In capitulo de verbo probat quod verbum 'secundum se dictum aliquid significet' et subiungit 'sed si est vel non est nondum significat', nec enim significat 'est', 'res esse vel non esse si <hoc> ipsum "est" purum dixeris: ipsum quoque nihil est etc.'."

39 An. Al., OHNEA, ed. Libera et Gazziero, pp. 345-346: "Secunda suppositio sequitur ex ista directe, quia si nomen non significat esse existentiae eius cuius est nomen, ut ostensum est, significat autem esse essentiae, sequitur quod esse essentiae et esse existentiae eius non sunt idem." Cf. Arist., Met. VII.5, 1031a12-13; cf. Auct. Arist. 1 n. 163, ed. Hamesse, 129, p. 80.

40 An. Al., OHNEA, ed. Libera et Gazziero, p. 346: "Quod autem significet esse essentiae eius patet quia significat idem quod sua definitio. [...] Item. Intellectus apprehendens rei essentiam non apprehendit necessario eius existentiam."

41 An. Al., OHNEA, ed. Libera et Gazziero, p. 349: "Item. [Ista propositio i)n se falsa est. Dicit enim Aristoteles quod 'oratio manens una et eadem numero mutatur a veritate in falsitatem secundum rei mutationem'. Cum ergo haec 'Caesar est' sit vera Caesare existente, illa re mutata manens eadem numero erit falsa. Non autem 
'homo' significa de manera unívoca al hombre que existe y al hombre que no existe, porque 'homo' significa al hombre sin considerar la existencia (sup. i) $\mathrm{y}$, en todo caso, siempre incluye la idea de un ser viviente en su significación. ${ }^{42}$ De este modo, la permanencia del sujeto se mantiene también en la predicación esencial, lo cual es sin duda más conveniente que la engorrosa idea de Bacon.

Por otra parte, la posición según la cual las palabras pierden su significación con la destrucción de las cosas es rechazada como 'cercana a la locura y más allá de toda locura' ${ }^{43}$ porque cualquiera que escuche una palabra que signifique algo que no existe, pero que conoce, la entiende. La posición de Bacon es entonces rechazada principalmente por incluír la existencia en el valor semiótico de las palabras.

Con respecto a la equivocidad de 'ser' con respecto al ser disposicional y al ser actual, An. Al. opone que 'est' representa o el ser actual (i.e., cuando es segundo adyacente) o el ser disposicional (i.e., cuando es tercero adyacente en la predicación esencial), pero nunca ambos al mismo tiempo y en la misma enunciación. 'Est' como tercero adyacente en la predicación esencial siempre representa una relación necesaria de inherencia entre los términos, y nunca la existencia de los términos, ${ }^{44}$ de modo que aunque 'Socrates est albus' incluya de manera implícita una afirmación de existencia, no es así del caso en 'homo est animal', porque en esta última 'est' sólo expresa que las nociones de hombre y de animal están relacionadas de manera necesaria, de modo que la noción de animal siempre es implicada por la noción de hombre, y esto sin importar si hay hombres o no. Con esta última afirmación An. Al. ataca tanto la posición de Olivi que sugiere que 'est' en 'homo est animal' puede

manet eadem numero nisi enuntiet idem numero et de eo eodem numero et, si ita sit, hoc nomen 'Caesar' univoce supponet in una et in alia."

42 An. Al., OHNEA, ed. Libera et Gazziero, p. 351: "Quod autem dicebatur primo quod hoc nomen 'homo' homine existente significat hominem vivum, nullo autem homine existente significat hominem mortuum, de quibus dicitur 'homo' aequivoce, modicam laut/ nullam habet proprietatem. Significat enim semper hominem vivum, quia impossibile est hominem significari vel intelligi sine vita, quia anima et vita sunt eadem. Si autem contingat hominem non esse vel mortuum esse, tamen hoc nomen non significat."

43 An. Al., OHNEA, ed. Libera et Gazziero, p. 342: "[...] sic videtur de ista positione quod sit similis dementiae et ultra omnes dementias, quia nec laicus, nec clericus, nec demens, nec sapiens in tantum egressus est quin nomine rei praeterita, quam cognovit, si ipsum audiat, moveatur in anima sua."

44 An. Al., OHNEA, ed. Libera et Gazziero, pp. 358-359: "Praeterea alii distinguunt istam 'omnis homo de necessitate est animal' eo quod hoc verbum 'est' potest dicere esse habituale vel esse actuale. Si dicat esse habituale dicunt quod est vera, si actuale falsa. Sed quia hoc verbum 'est' secundum adiacens semper copulat rem suam quae est existere, quando autem est tertium adiacens copulat rem illius cui apponitur, credo quod possit copulare esse habituale vel actuale, nunquam tamen in eadem propositione. Sed quandocumque praedicatur secundum adiacens copulat esse actuale, quandocumque autem praedicatur tertio adiacens cum praedicato essentiali copulat esse habituale, nec est copulare esse habituale dicere extrema esse habitu vel in potentia, sicut quidam credunt. Sed esse habituale appellabant antiqui dicere habitudinem vel inhaerentiam extremorum, et non existentiam eorum. Dicere autem esse actuale est dicere eorum existentiam." 
eventualmente indicar existencia actual, como la posición de Boecio según la cual 'est' siempre indica existencia actual.

Debe notarse, sin embargo, que la afirmación aristotélica de que los onomata y los rhêmata no afirman existencia, sólo es válida cuando éstos están por fuera de un contexto proposicional, y por lo tanto el supuesto ii) de An. Al. no concierne los términos nominales (onomata) y los términos atribuídos (rhêmata) que ya son sujetos y predicados de una enunciación. Boecio de Dacia, por ejemplo, aceptaría que 'homo', en cuanto nombre, no afirma la existencia del hombre, pero que la verdad de 'homo est animal' sí afirma la existencia del hombre, porque la afirmación de la existencia viene de la composición indicada por 'est' y no de la significación de los términos. ${ }^{45}$

Después de estas objeciones, An. Al. presenta su propia solución del sofisma, diciendo que 'OHNEA' es verdadera simplemente, cuya prueba es dada en el argumento A. ${ }^{46}$ Recordemos, sin embargo, que este argumento presupone la existencia de por lo menos un supuesto del hombre, que es sin embargo lo que el sofisma niega por hipótesis. Finalmente, al conceder este argumento, An. Al. rechaza al mismo tiempo la multiplicidad de las formas, y afirma que, en oposición a la especie que agrega una diferencia al género, la especie y el individuo tienen exactamente la misma forma. Como los nombres son impuestos gracias al entendimiento de una forma, los nombres de e.g. los individuos del hombre - nombres propios - son impuestos gracias al entendimiento de la forma del hombre, de modo que 'Socrates' y 'homo' significan exactamente la misma esencia - la esencia del hombre - pero de manera diferente. Por consiguiente, predicar 'homo' de 'Socrates' es predicar lo mismo sobre lo mismo, de modo que 'Socrates est homo' es tan necesaria como 'homo est homo' e incluso más necesaria que 'homo est animal' ${ }^{47}$

45 Cf. P. Crivelli, 2004, ch. 5.2, quien sostiene una posición cercana a la de Boecio. La posición opuesta es tomada por P. Thom, 2002, pp. 293-301.

46 Ver argumento A, en la sección 1.

47 An. Al., OHNEA, ed. Libera et Gazziero, pp. 361-362: "Et iterum individuum non addit formam aliquam supra speciem vel differentiam nec habet aliam formam a forma speciei et hoc est quod scribitur quod 'species est totum esse individuorum'; genus autem non est totum esse speciei quia species addit differentiam et esse supra genus, quae differentia non est in genere nisi potestate solum. Nomen autem imponitur a forma, ergo cum individuum non habeat aliam formam a forma speciei, ut dictum est, ab eadem forma et ad eandem <designandam > imponitur nomen speciei et individui; idem ergo significant hoc homen 'Sor' et hoc nomen 'homo' modo solum alio, qui modus non est accidentalis, [...]; ergo praedicare hominem de Sorte est praedicare idem de se, et qui negat talem praedicationem idem affirmat et negat, sicut visum fuit prius. Haec ergo 'Sor est homo' non solum est necessaria immo magis necessaria quam ista 'homo est animal', et aeque necessaria ut ista 'homo est homo'." 
El sofisma An. Al. es sin duda un documento histórico excepcional a la hora de reconstruír la discusión sobre la relación entre la significación y la referencia vacía en la segunda mitad del siglo 13. Su solución y su tratamiento de las posiciones alternativas, sin embargo, evidencian cierta dificultad en su evaluación sobre el alcance de la lógica aristotélica en lo que concierne a las afirmaciones con sujetos vacíos. Las nociones de significación y reimposición, que son suficientes para que Bacon explique tanto la dimensión significativa como la dimensión referencial del lenguaje, son sin duda un tanto traídas de los cabellos y justamente criticadas. Pero Olivi y Boecio de Dinamarca presentan dos ideas de gran interés que son pasadas por alto en el sofisma anónimo: primero, la idea de Olivi de que todas las palabras, la cópula 'est' incluída, son susceptibles de usos diferentes, sumada a la cuidadosa aclaración por parte de Olivi de que el ser disposicional y el ser actual no representan dos categorías ontológicas diferentes sino dos aproximaciones cognitivas diferentes a una misma cosa, aproximaciones que implican dos usos diferentes de 'est'; segundo, el análisis impecable, desde el punto de vista de la lógica aristotélica, que Boecio de Dinamarca nos presenta en su tratamiento de este sofisma, donde desplaza la noción de significación al campo semiótico y nota, de manera correcta en mi opinión, que la cuestión de las condiciones de verdad y falsedad de las afirmaciones y la cuestión de la significación de las palabras tienen poco que ver la una con la otra.

\section{Referências}

BÄCK, A. “Aristotle's Theory of Predication”. Leiden: E. J. Brill, 2000.

BACON, R. "De signis”. In: K. M. Fredborg, L. Nielsen, J. Pinborg (eds.), 1978. pp. 75-136.

BOUREAU, A.; PIRON, S. (eds.). "Pierre de Jean Olivi (1248/1298). Pensée scolastique, dissidence spirituelle et société". Paris: Vrin, 1999.

BOS, E. P. (ed.). "Medieval Logic and Semantics". Studies Dedicated to L. M. de Rijk... on the Occasion of His 60th Birthday. Leiden/Nijmegen: Ingenium Publishers, 1981.

BRAAKHUIS et al. (eds.). "English Logic and Semantics: From the End of the Twelfth Century to the Time of Ockham and Burleigh". Acts of the $4^{\text {th }}$ European on Mediaeval Logic and Semantics, Leiden-Nijmegen, 23-27 April 1979. Brepols, 1979. CASTON, V.; GRAHAM, D. W. (eds.). "Presocratic Philosophy: Essays in Honour of Alexander Mourelatos". Aldershot: Ashgate, 2002.

CESALLI, L., DE LIBERA, A., GOUBIER, F. "Does Loving Every Mean Loving Every Every, even Non-existent Ones? Distribution and Universals in the Opus puerorum". In: J. Fink, H. Hansen \& A.M. Mora-Márquez (eds.), 2013. pp. 305-336. 
CRIVELLI, P. “Aristotle on Truth". Cambridge: CUP, 2004.

DE LIBERA, A. "Faire de nécessité loi: Théories de la modalité dans le sophisma 'Omnis homo de necessitate est animal' du codex parisisnus 16135, ff. 11rb-12rb". AHDLMA, 76, pp. 179-233, 2009.

"Omnis homo de necessitate est animal. Référence et modalité selon l'Anonymus Erfordensis Q. 328 (Pseudo-Robert Kilwardby)". AHDLMA, 69, pp. 201237, 2002.

"La référence vide". Paris: PUF, 2002.

"Référence et quantification: Sur la théorie de la distributio au XIIIe siècle". In: A. de Libera, A. Elamrani-Jamal, A. Galonnier (eds.), 1997. pp. 177-200. "César et le phénix: Distinctiones et sophismata parisiens du XIIIe siècle". Pisa: Centro di cultura medievale della Scuola Normale Superiore, 1991.

. "Roger Bacon et la référence vide. Sur quelques antécédents médiévaux du paradoxe de Meinong". In: J. Jolivet et al. (eds.), 1991. pp. 85-120.

DE LIBERA, A., GAZZIERO, L. "Le Sophisma 'Omnis homo de necessitate est animal' du Parisinus Latinus 16135, ff. 11rb-12rb”. AHDLMA, 75, Vol. 1, pp. 323368, 2008.

DE PARIS, N. "Syncategoremata”. Ed. H. A. G. Braakhuis, Meppel: Krips Repro, 1979.

DE SHERWOOK, G. “Syncategoremata”. Ed. J. R. O’Donnell. Mediaeval Studies, 3, pp. 46-93, 1941.

EBBESEN, S. "The Man who Loved Every: Boethius of Dacia on logic and metaphysics". The Modern Schoolman, 82, pp. 235-250, 2005.

. "Boethius of Dacia: Science is a serious game". Theoria, 66, pp. 145-158, 2000.

EBBESEN, S., GOUBIER, F. “A Catalogue of $13^{\text {th }}$ Century Sophismata”. Paris: Vrin, 2010.

ESPAÑA, P. "Syncategoremata". Ed. L. M. de Rijk, Leiden: E. J. Brill, 1992.

. "Tractatus". Ed. L. M. de Rijk. Assen: Van Gorcum, 1972.

FINK, J., HANSEN, H., MORA-MÁRQUEZ, A. M. (eds.). "Logic and Language in the Middle Ages. A Volume in Honour of Sten Ebbesen". Leiden: E. J. Brill, 2013.

FREDBORG, K. M., NIELSEN, L.; PINBORG, J. (eds.). "An Unedited Part of Roger Bacon's Opus maius: De signis". Traditio, 34, 1978.

GOUBIER, F. "Les syncatégorèmes au XIIIe siècle". Histoire Epistémologie Langage, 25, Vol. 2, pp. 85-113, 2003.

"Influences prédicatives et conséquences reférentielles: un aspect de l'approche terministe de la première moitié du XIIIe siècle". CIMAGL, 71, pp. 37-70, 2000.

JOLIVET, J. et al. (eds.). "Lectionum varietates. Hommage à Paul Vignaux (1904/1987)”. pp. 85-120. Paris: Vrin, 1991.

"Roger Bacon et le problème de l'appellatio univoca". In: Braakhuis et al. (eds.), 1981. pp. 193-221. 
KNUUTTILA, S. "Modalities in Medieval Philosophy". London, New York: Routledge, 1993.

LAGERLUND, H. (ed.). "Encyclopedia of Medieval Philosophy". pp. 1229-1236. Berlin: Springer, 2011.

MALONEY, T. S. "Roger Bacon on Equivocation”. Vivarium, 22, pp. 85-112, 1984.

MÁRQUEZ, A. M. "Martinus Dacus and Boethius Dacus on the Signification of Terms and the Truth-value of Assertions". Vivarium, 52, pp. 23-48, 2014.

. "Pragmatics in Peter John Olivi's Account of Signification of Common Names". Vivarium, 49, pp. 150-164, 2011.

NOVAES, C. D. "Medieval Theories of Supposition”. In: H. Lagerlund (ed.), 2011. pp. 1229-1236.

OLIVI, P. J. “Quaestiones logicales”. Ed. S. F. Brown. Traditio, 42, pp. 335-388, 1986.

READ, S. (ed.). "Sophisms in Medieval Grammar and Logic". Dordrecht: Kluwer, 1993.

SANTOS, E. P. "Mapeando argumentos no sofisma 'omnis homo de necessitate est animal"". Dois Pontos, 7.1, 2010.

TABARRONI, A. “'Ominis phoenix est: quantification and existence in a new sophismata collection (MS Clm 14522)". In: S. Read (ed.), 1993, pp. 185-201.

THOM, P. “On the Pervasiveness of Being”. In: V. Caston, D.W. Graham (eds.), 2002. pp. 293-301. 